\title{
Caries Increment and Salivary Microbiome during University Life: A Prospective Cohort Study
}

\author{
Yoko Uchida-Fukuhara ${ }^{1,2,3, *}$, Daisuke Ekuni ${ }^{4}{ }^{(}$, , Md Monirul Islam ${ }^{4}\left({ }^{\circ}\right.$, Kota Kataoka ${ }^{2,4}$, \\ Ayano Taniguchi-Tabata ${ }^{1}{ }^{\circledR}$, Daiki Fukuhara ${ }^{1}$, Naoki Toyama ${ }^{4}{ }^{\circledR}$, Terumasa Kobayashi ${ }^{4}$, \\ Kohei Fujimori ${ }^{4}$, Nanami Sawada ${ }^{4}$, Yoshiaki Iwasaki ${ }^{5}$ and Manabu Morita ${ }^{4}$ \\ 1 Department of Preventive Dentistry, Okayama University Hospital, Okayama 700-8558, Japan; \\ de19026@s.okayama-u.ac.jp (A.T.-T.); de20041@s.okayama-u.ac.jp (D.F.) \\ 2 Advanced Research Center for Oral and Craniofacial Sciences, Okayama University Dental School, \\ 2-5-1 Shikata-cho, Kita-ku, Okayama 700-8558, Japan; de18017@s.okayama-u.ac.jp \\ 3 Department of Oral Morphology, Graduate School of Medicine, Dentistry and Pharmaceutical Sciences, \\ Okayama University, Okayama 700-8558, Japan \\ 4 Department of Preventive Dentistry, Okayama University Graduate School of Medicine, Dentistry and \\ Pharmaceutical Sciences, Okayama 700-8558, Japan; dekuni7@md.okayama-u.ac.jp (D.E.); \\ p3a99o50@s.okayama-u.ac.jp (M.M.I.); pu171qxi@s.okayama-u.ac.jp (N.T.); \\ de421015@s.okayama-u.ac.jp (T.K.); k-fujimori@okayama-u.ac.jp (K.F.); de422027@s.okayama-u.ac.jp (N.S.); \\ mmorita@md.okayama-u.ac.jp (M.M.) \\ 5 Health Service Center, Okayama University, Okayama 700-8530, Japan; yiwasaki@okayama-u.ac.jp \\ * Correspondence: de20006@s.okayama-u.ac.jp; Tel.: +81-86-235-6712
}

Received: 24 March 2020; Accepted: 21 May 2020; Published: 25 May 2020

\begin{abstract}
The purpose of this 3-year prospective cohort study was to explore the relationship between an increase in dental caries and oral microbiome among Japanese university students. We analyzed 487 students who volunteered to receive oral examinations and answer baseline (2013) and follow-up (2016) questionnaires. Of these students, salivary samples were randomly collected from 55 students at follow-up and analyzed using next-generation sequencing. Students were divided into two groups: increased group ( $\triangle$ decayed, missing, and filled teeth $(\triangle \mathrm{DMFT})$ score increased during the 3-year period) and non-increased group ( $\triangle$ DMFT did not increase). Thirteen phyla, 21 classes, 32 orders, 48 families, 72 genera, and 156 species were identified. Microbial diversity in the increased group $(n=14)$ was similar to that in the non-increased group $(n=41)$. Relative abundances of the family Prevotellaceae $(p=0.007)$ and genera Alloprevotella $(p=0.007)$ and Dialister $(p=0.039)$ were enriched in the increased group compared with the non-increased group. Some bacterial taxonomic clades were differentially present between the two groups. These results may contribute to the development of new dental caries prevention strategies, including the development of detection kits and enlightenment activities for these bacteria.
\end{abstract}

Keywords: salivary microbiome; sequence analysis; young adult; dental caries; saliva; oral health

\section{Introduction}

Dental caries caused by some pathogens of the oral microbiome is a widespread disease. In the Global Burden of Disease 2015 Study, untreated caries in permanent teeth was the most prevalent condition, affecting 2.5 billion people worldwide [1]. The cause of dental caries is multifactorial. In addition to major microbial species mutans streptococci (predominantly Streptococcus mutans and S. sobrinus), physical, biological, environmental, behavioral, and lifestyle-related factors are risk factors for dental caries [2]. 
Recently, next-generation DNA sequencing (NGS) analyses have attracted attention for use in microbiome studies, including dental caries. A pyrosequencing (NGS analysis) study revealed genera Prevotella, Lactobacillus, and Streptococcus were increased in carious dentin compared with healthy dentin [3]. Another study revealed that genera Alloprevotella, Atopobium, Lautropia, Megasphaera, Selenomonas, and Veillonella were enriched in saliva from a high-risk caries group [4]. According to the ecological hypothesis of dental caries [5], the proportion of acidogenic and aciduric taxa, such as mutans streptococci and Lactobacillus, are increased and lead to demineralization. In addition, this hypothesis suggests that amino acid-degrading microbial taxa, including Prevotella, and Fusobacterium species may also cause the acidic environment required for demineralization $[5,6]$. These results indicate that both mutans and non-mutans streptococci may contribute to dental caries. However, many caries studies using sequencing analyses targeted young children with primary dentition, and few studies have focused on young adults and older people [4,7-10]. Whether a relationship between the increase in dental caries and oral microbiome exists in these latter populations remains unclear.

In Japan, there remains a high caries prevalence in young adults. Among young adults (20-24 years), $79.4 \%$ had caries experience [11]. Thus, it is crucial to prevent dental caries in the general population.

Therefore, we hypothesized that an increase in dental caries during university life is associated with a unique oral microbiome. The purpose of this 3-year prospective cohort study was to explore the relationship between an increase in caries and oral microbiome among Japanese university students. In addition, we investigated the association between other factors and caries increment.

\section{Materials and Methods}

\subsection{Study Population}

The inclusion criteria were Japanese students who volunteered to receive oral examinations at the Health Service Center of Okayama University both in April 2013 (baseline) and April 2016 (follow-up). We excluded students who provided incomplete responses in their questionnaires.

\subsection{Ethical Procedures and Informed Consent}

All study protocols were approved by the ethics committee of Okayama University Graduate School of Medicine, Dentistry, and Pharmaceutical Sciences and Okayama University Hospital (no. 1060). All targeted participants gave their informed verbal consent for study participation. This study followed the strengthening the reporting of observational Studies in Epidemiology (STROBE) guidelines.

\subsection{Questionnaire}

At baseline, students answered questions concerning age, sex, systemic diseases, and oral health behaviors (daily frequency of tooth brushing, use of dental floss, and visits to dental clinics for regular checkups) [12]. Furthermore, at follow-up, four additional questions were included about fluoride dentifrices, knowledge of the effectiveness of fluoride, daily frequency of eating sweets, and smoking during university life.

\subsection{Oral Examination}

Oral examinations were performed by five calibrated dentists (Daisuke Ekuni, Kota Kataoka, Mayu Yamane-Takeuchi, Shinsuke Mizutani, and Tetsuji Azuma). After counting the number of teeth, the oral hygiene state was evaluated using the Debris Index-Simplified (DI-S) score [13]. The decayed, missing, and filled teeth (DMFT) scores was recorded according to the World Health Organization criteria [14]. After the theoretical training, to assess intra- and inter-examiner agreement, DMFT scores were recorded and repeated within a 2-week interval in two volunteers. Data were analyzed using a non-parametric kappa test. The kappa values were $>0.8$. 


\subsection{DNA Extraction and NGS Analysis}

At follow-up, unstimulated saliva $(>1 \mathrm{~mL})$ was randomly collected into sterile ice-chilled $15 \mathrm{~mL}$ tubes from students (from 09:00 to 16:00) before the dental examination and frozen at $-80{ }^{\circ} \mathrm{C}$ until analysis. To prevent possible protein dilution, unstimulated saliva was collected instead of stimulated saliva [15-17]. A random number list was used for selecting participants for saliva collection. The saliva of selected participants was not collected at baseline. Saliva DNA was collected using the QIAamp DNA Mini Kit (Qiagen, Hilden, Germany) according to the manufacturer's instructions using sterile equipment and DNA removal reagents for laboratory instruments. Collected DNA was stored at $-20{ }^{\circ} \mathrm{C}$ for further analysis. For amplified bacterial DNA, V3 and V4 regions of the 16S rRNA gene were amplified using primers 357F (5'-TCGTCGGCAGCGTCAGATGT GTATAAGAGACAGCCTACGGGNGGCWGCAG-3') and 781R (5'-GTCTCGTGGGCTCGGAGATG TGTATAAGAGACAGGACTACHVGGGTATCTAATCC-3') at Okayama University Hospital Biobank (Okayama University Hospital, Okayama, Japan) according to the standard protocol using the MiSeq platform (MiSeq Reagent V3 600 cycles, Illumina, San Diego, CA, USA). Quality of raw sequence reads was checked using FastQC (version 0.11.3, Babraham Bioinformatics, Cambridge, UK) and analyzed using USEARCH (version 8.0.1623, https://www.drive5.com/usearch/) at the Oral Microbiome Center (Taniguchi Dental Clinic, Kagawa, Japan). After removing chimeric reads, duplicated reads, and short reads $<400 \mathrm{bp}$, preprocessed reads of each sample were clustered into operational taxonomic units (OTUs) at 97\% level of nucleotide similarity using UCLUST algorithm to determine the number of OTUs. Furthermore, these reads were analyzed to identify human oral taxa using the Human Oral Microbiome Database (version 14.5; http://www.homd.org/).

\subsection{Statistical Analyses}

We estimated the sample size for the saliva examination based on a previous study [4] using G*Power (version 3.1.9.4, Düsseldorf, Germany). The difference in $\alpha$ diversity (Simpson index; caries group: $0.075 \pm 0.019$, healthy group: $0.099 \pm 0.037$ ) was selected as the primary outcome [4]. To calculate the effect size, mean and standard deviation of $0.024 \pm 0.018$ was considered to detect a difference in $\alpha$ diversity between the two communities. Based on the data, the minimum sample size required was 52 to provide a power of $91 \%$ with an alpha of 0.05 by $t$-test. Participants were divided into two groups based on the change in DMFT score during the 3-year follow-up period; participants with $\triangle \mathrm{DMFT}>0$ were categorized into the "increased group," while participants with $\triangle \mathrm{DMFT}=0$ were categorized into the "non-increased group" [18]. The normality of the data was confirmed by the histogram and quantile-quantile plot. The Mann-Whitney U test and Fisher's exact test were used to determine the presence of significant differences in variables of oral examination and questionnaire between increased and non-increased groups. Associations between variables and dental caries were examined in a series of logistic regression models, and the odds ratio (OR) and $95 \%$ confidence interval (CI) were calculated. Logistic regression models were reviewed for goodness-of-fit and validated using the Hosmer-Lemeshow statistic. A $p$-value $<0.05$ was considered significant. Statistical analyses were performed using SPSS (version 25.0; IBM, Tokyo, Japan).

Alpha diversity was determined using R (version 3.4.3; The R Project for Statistical Computing, http://www.R-project.org). The species richness of saliva microbiota of individuals was measured by Chao1 and the abundance-based coverage estimator (ACE) indices. The diversity of the saliva microbiota was measured by Shannon and Simpson indices. Microbial beta diversity was visualized by principal coordinates analysis using the Calypso software tool (http://bioinfo.qimr.edu.au/calypso/). The Mann-Whitney $U$ test was used to assess significant differences in the abundance of taxa between increased and non-increased groups. Rarefaction curves were calculated using Calypso to compare microbial richness among samples. To compare the microbial composition between increased and non-increased groups, relative abundances (\%) were calculated from the taxonomic abundance count divided by preprocessed reads. Linear discriminant analysis (LDA) effect size (LEfSe) methods were used to identify taxa with differentiating relative abundance using the online interface Galaxy 
(http://huttenhower.sph.harvard.edu/lefse/). The threshold for the logarithmic LDA score for biomarker discovery was set at $2.0[19,20]$.

\section{Results}

\subsection{Participant Characteristics}

The study flowchart is shown in Figure 1; 293 students (follow-up rate: 13.4\%; 149 males, 144 females; mean age and standard deviation at baseline: $18.2 \pm 0.77$ years) were analyzed (Table 1 ). At follow-up, the DI-S score of selected students $(n=55)$ was significantly lower than that of all participants $(n=293 ; p<0.05, t$-test). No significant differences were observed in the other parameters evaluated between selected students and all participants $(p<0.05$, Mann-Whitney $U$ test and Fisher's exact test).

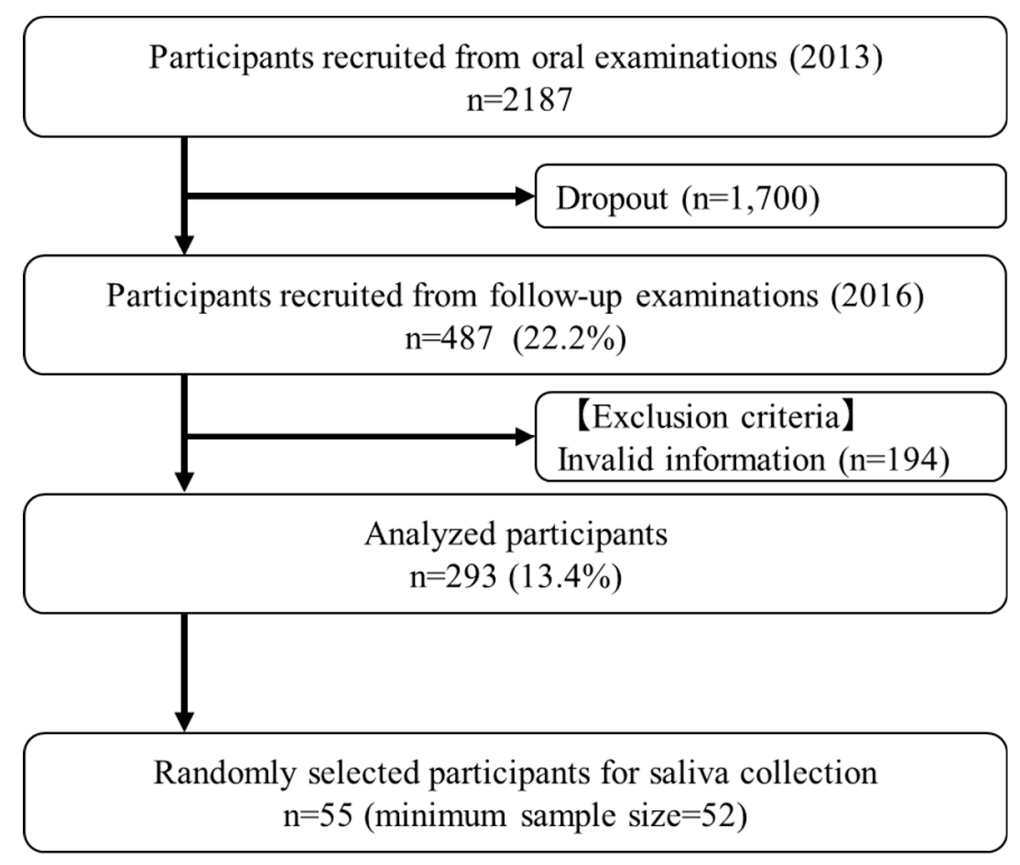

Figure 1. Flowchart of study participants. This flowchart shows the process for selecting analyzed participants. At baseline, 2187 students received the oral examination. Because follow-up oral examination was not mandatory, only 487 students received a second oral examination. Of these students, 55 students were randomized and consented to saliva collection.

Table 1. Oral condition and health behaviors in total and selected participants at baseline.

\begin{tabular}{|c|c|c|c|c|c|}
\hline \multirow[t]{2}{*}{ Variable } & & & Total Participants & $\begin{array}{c}\text { Selected } \\
\text { Participants }\end{array}$ & \multirow[t]{2}{*}{$p$-Value ${ }^{3}$} \\
\hline & & & $(n=293)$ & $(n=55)$ & \\
\hline \multirow{8}{*}{ Baseline } & Gender & Male/Female & $149 / 144(50.9 / 49.1)^{1}$ & $17 / 38(30.9 / 69.1)$ & 0.006 \\
\hline & Age (year) & & $18.22 \pm 0.76^{2}$ & $18.24 \pm 0.47$ & 0.378 \\
\hline & $\begin{array}{l}\text { Number of teeth } \\
\text { present }\end{array}$ & & $28.49 \pm 1.43$ & $28.42 \pm 1.49$ & 0.699 \\
\hline & DMFT score & & $1.00 \pm 2.01$ & $1.44 \pm 2.79$ & 0.459 \\
\hline & DI-S & & $0.41 \pm 0.37$ & $0.33 \pm 0.32$ & 0.363 \\
\hline & \multirow{3}{*}{$\begin{array}{l}\text { Daily frequency of } \\
\text { tooth brushing }\end{array}$} & 1 & $55(18.8)$ & $9(16.4)$ & \multirow{3}{*}{0.914} \\
\hline & & 2 & $212(72.4)$ & $41(74.5)$ & \\
\hline & & 3 & $26(8.9)$ & $5(9.1)$ & \\
\hline
\end{tabular}


Table 1. Cont.

\begin{tabular}{|c|c|c|c|c|c|}
\hline Variable & & & Total Participants & $\begin{array}{c}\text { Selected } \\
\text { Participants }\end{array}$ & $p$-Value ${ }^{3}$ \\
\hline \multirow{2}{*}{ Baseline } & Use of dental floss & yes & $20(6.8)$ & $5(9.1)$ & 0.551 \\
\hline & $\begin{array}{l}\text { Regular dental } \\
\text { check-ups }\end{array}$ & yes & $36(12.3)$ & $13(23.6)$ & 0.034 \\
\hline \multirow{15}{*}{ Follow-up } & $\begin{array}{l}\text { Number of teeth } \\
\text { present }\end{array}$ & & $29.2 \pm 1.77$ & $28.9 \pm 1.78$ & 0.353 \\
\hline & DMFT score & & $1.70 \pm 2.99$ & $1.82 \pm 2.97$ & 0.477 \\
\hline & DI-S & & $0.81 \pm 2.49$ & $0.31 \pm 0.36$ & 0.037 \\
\hline & \multirow{3}{*}{$\begin{array}{l}\text { Daily frequency of } \\
\text { tooth brushing }\end{array}$} & 1 & $45(15.4)$ & $8(14.5)$ & \multirow{3}{*}{0.323} \\
\hline & & 2 & $220(75.1)$ & $38(69.1)$ & \\
\hline & & 3 & $28(9.6)$ & $9(16.4)$ & \\
\hline & Use of dental floss & yes & $45(15.4)$ & $12(21.8)$ & 0.237 \\
\hline & $\begin{array}{l}\text { Regular dental } \\
\text { check-ups }\end{array}$ & yes & $41(14.0)$ & $11(20.0)$ & 0.301 \\
\hline & Current smoker & yes & $9(3.07)$ & $2(3.36)$ & \\
\hline & $\begin{array}{l}\text { Use of fluoride } \\
\text { containing paste }\end{array}$ & yes & $155(52.9)$ & $28(50.9)$ & 0.883 \\
\hline & $\begin{array}{l}\text { Knowledge of the } \\
\text { effectiveness of fluoride }\end{array}$ & yes & $250(85.3)$ & $46(83.6)$ & 0.686 \\
\hline & \multirow{4}{*}{$\begin{array}{l}\text { Frequency of sweet } \\
\text { intake (daily) }\end{array}$} & 0 & $53(18.1)$ & $8(14.5)$ & \multirow{4}{*}{0.295} \\
\hline & & 1 & $163(55.6)$ & $37(67.3)$ & \\
\hline & & 2 & $56(19.1)$ & $9(16.4)$ & \\
\hline & & 3 & $21(7.2)$ & $1(1.8)$ & \\
\hline
\end{tabular}

DMFT, decayed, missing, and filled teeth score; DI-S, debris index-simplified index; ${ }^{1}$ Data are expressed as $n(\%)$;

${ }^{2}$ Data are expressed as mean \pm standard deviation; ${ }^{3}$ Fisher's exact test or Mann-Whitney U test.

In all participants, DMFT scores, DI-S score at baseline, and the percentage of students who had knowledge of fluoride at follow-up were significantly higher in the increased group than those in the non-increased group $(p<0.05$; Table 2$)$. In the logistic regression analysis, increase in dental caries was significantly related to DMFT score (OR: 1.191, 95\% CI: 1.033-1.373, $p=0.016)$ and DI-S score (OR: 3.6, 95\% CI: 1.355-6.913, $p=0.007$ ) at baseline.

\subsection{Salivary Microbiome Analysis}

In our study, 3,195,127 quality-filtering reads $(58,093 \pm 16,532)$ from 55 saliva samples were used for analysis. A total of 196 OTUs were obtained from saliva samples. Of these, 13 phyla, 21 classes, 32 orders, 48 families, 72 genera, and 156 species were identified. There were no significant differences in the number of each taxonomic level between increased and non-increased groups $(p \geq 0.05)$. Furthermore, there were no significant differences in the species richness of individuals between increased and non-increased groups (Figure 2a; $p \geq 0.05$ ). Moreover, no significant differences were observed in species diversity and bacterial communities between the two groups (Figure $2 b, c ; p \geq 0.05$ ). 
Table 2. Differences in dental caries-related variables between increased and non-increased groups.

\begin{tabular}{|c|c|c|c|c|c|c|c|c|}
\hline \multirow{2}{*}{ Variable } & & & \multicolumn{3}{|c|}{ Total Participants $(n=293)$} & \multicolumn{3}{|c|}{ Selected Participants $(n=55)$} \\
\hline & & & $\begin{array}{c}\text { Non-Increased } \\
(n=220)\end{array}$ & $\begin{array}{c}\text { Increased } \\
(n=73)\end{array}$ & $p$-Value ${ }^{3}$ & $\begin{array}{c}\text { Non-Increased } \\
(n=41)\end{array}$ & \multirow{2}{*}{$\begin{array}{c}\text { Increased } \\
(n=14) \\
\end{array}$} & $p$-Value \\
\hline \multirow{9}{*}{ Baseline } & & & & & & & & \\
\hline & Number of teeth present & & $28.37 \pm 0.37^{1}$ & $28.82 \pm 1.65$ & 0.017 & $28.27 \pm 1.28$ & $28.86 \pm 1.95$ & 0.127 \\
\hline & DMFT score & & $0.78 \pm 0.12$ & $1.68 \pm 2.35$ & $<0.001$ & $1.32 \pm 2.64$ & $1.79 \pm 3.28$ & 0.71 \\
\hline & DI-S & & $0.38 \pm 0.35$ & $0.52 \pm 0.42$ & 0.008 & $0.33 \pm 0.29$ & $0.43 \pm 0.41$ & 0.619 \\
\hline & \multirow{3}{*}{ Daily frequency of tooth brushing } & 1 & $38(17.3)^{2}$ & $17(23.3)$ & \multirow{3}{*}{0.452} & $8(19.5)$ & $1(7.1)$ & \multirow{3}{*}{0.504} \\
\hline & & 2 & $161(73.2)$ & $51(69.9)$ & & $29(65.9)$ & $12(85.7)$ & \\
\hline & & 3 & $21(9.5)$ & $5(6.8)$ & & $4(9.8)$ & $1(7.1)$ & \\
\hline & Use of dental floss & yes & $15(6.8)$ & $5(6.8)$ & 0.993 & $2(4.9)$ & $3(21.4)$ & 0.063 \\
\hline & Regular dental check-ups & yes & $23(10.5)$ & $13(17.8)$ & 0.097 & $9(22)$ & $4(28.6)$ & 0.615 \\
\hline \multirow{15}{*}{ Follow-up } & Number of teeth present & & $29.22 \pm 1.77$ & $29.36 \pm 1.82$ & 0.323 & $28.78 \pm 1.74$ & $29.29 \pm 1.93$ & 0.371 \\
\hline & DMFT score & & $0.78 \pm 0.12$ & $4.26 \pm 3.98$ & $<0.001$ & $1.32 \pm 2.64$ & $3.28 \pm 3.49$ & $<0.001$ \\
\hline & DI-S & & $0.94 \pm 2.87$ & $0.43 \pm 0.42$ & 0.932 & $0.33 \pm 0.37$ & $0.27 \pm 0.37$ & 0.372 \\
\hline & \multirow{3}{*}{ Daily frequency of tooth brushing } & 1 & $12(15.4)$ & $34(15.4)$ & \multirow{3}{*}{0.522} & $7(17.8)$ & $1(7.1)$ & \multirow{3}{*}{0.522} \\
\hline & & 2 & $61(78.2)$ & $164(73.9)$ & & $27(65.9)$ & $11(78.6)$ & \\
\hline & & 3 & $5(6.4)$ & $24(10.8)$ & & $7(17.1)$ & $2(14.3)$ & \\
\hline & Use of dental floss & yes & 30 (13.6) & $15(20.5)$ & 0.156 & $8(19.5)$ & $4(28.6)$ & 0.479 \\
\hline & Regular dental check-ups & yes & $29(13.2)$ & $12(16.4)$ & 0.487 & $8(19.5)$ & $3(21.4)$ & 0.877 \\
\hline & Current smoker & yes & $7(3.2)$ & $2(2.7)$ & 0.982 & $1(2.4)$ & $1(7.1)$ & 0.448 \\
\hline & Use of fluoride containing paste & yes & $116(52.7)$ & $39(53.4)$ & 0.918 & $18(43.9)$ & $10(71.4)$ & 0.075 \\
\hline & Knowledge of the effectiveness of fluoride & yes & $182(82.7)$ & $68(93.2)$ & 0.029 & $34(82.9)$ & $12(85.7)$ & 0.808 \\
\hline & \multirow{4}{*}{ Frequency of sweet intake (daily) } & 0 & 41 (18.6) & $12(16.4)$ & \multirow{4}{*}{0.769} & $7(17.1)$ & $1(7.1)$ & \multirow{4}{*}{0.693} \\
\hline & & 1 & $124(56.4)$ & $39(53.4)$ & & $29(63.4)$ & $11(78.6)$ & \\
\hline & & 2 & 39 (17.7) & $17(23.3)$ & & $7(17.1)$ & $2(14.3)$ & \\
\hline & & 3 & $16(7.3)$ & $5(6.8)$ & & $1(2.4)$ & $0(0.0)$ & \\
\hline
\end{tabular}

The increased group was defined as $\triangle \mathrm{DMFT}>0$ during the 3-year follow-up period; The non-increased group was defined as $\triangle \mathrm{DMFT}=0$ during the 3-year follow-up period; DMFT: decayed, missing, and filled teeth; DI-S, debris index-simplified. ${ }^{1}$ Data are expressed as mean \pm standard deviation. ${ }^{2}$ Data are expressed as $n(\%) .{ }^{3}$ Fisher's exact test or Mann-Whitney $\mathrm{U}$ test. 
(a)

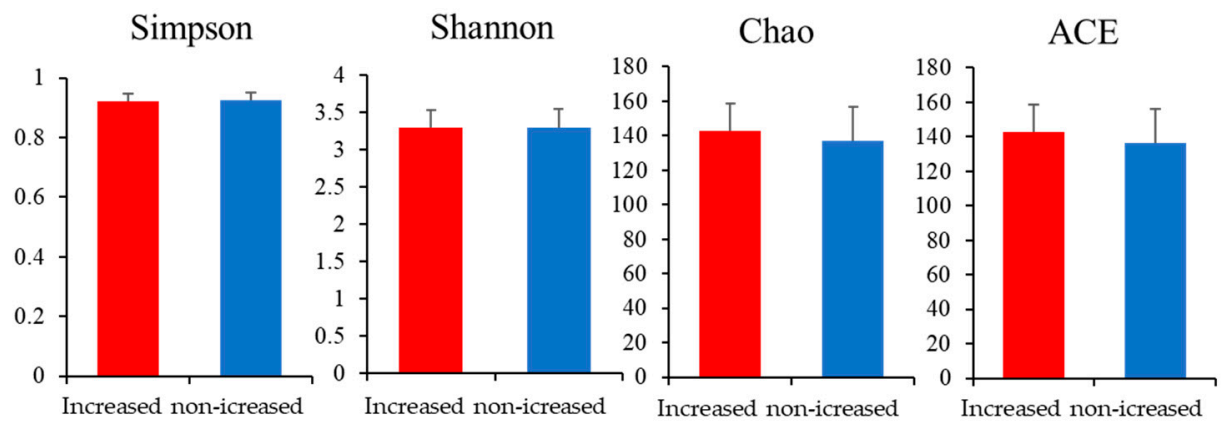

(b)

(c)

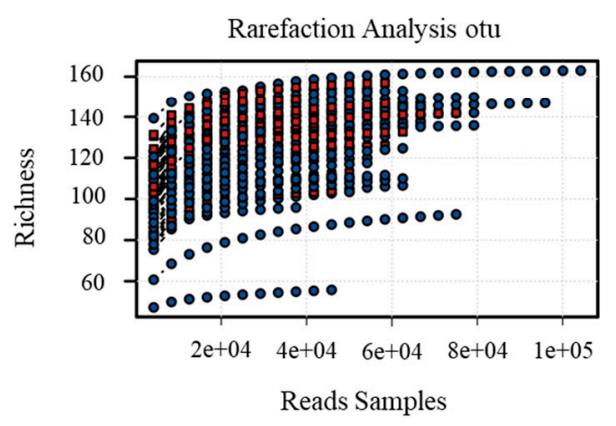

PCoA Bray-Curtis OTU

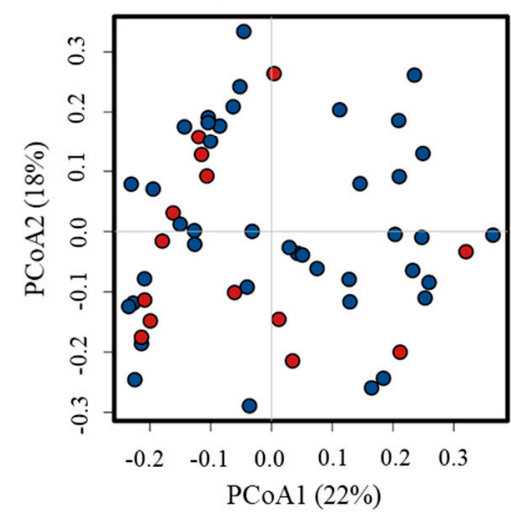

Figure 2. Comparison of the oral microbiome diversity between increased and non-increased groups. (a) Saliva microbiome diversity between increased (red) and non-increased groups (blue) was compared. Alpha diversity metrics for Simpson, Shannon, Chao1, and ACE indices were calculated and illustrated by box plots. There were no significant differences in diversity between increased and non-increased groups ( $t$-test, $p \geq 0.05$ ). (b) Rarefaction curves of increased (red) and non-increased (blue) groups based on the observed operational taxonomic unit (OTU). There were no significant differences in species richness between increased and non-increased groups ( $t$-test, $p \geq 0.05$ ). (c) Principal coordinates analysis (PCoA) based on the Bray-Curtis index. Increased (red) and non-increased (blue) groups did not tend to separate.

\subsection{Microbial Composition}

There were no significant differences in microbial composition at class and order levels between increased and non-increased groups. At the phylum level, the increased group had a higher population of Proteobacteria than the non-increased group (Table $3 ; p=0.029$ ). At the family level, the increased group had a higher population of Prevotellaceae than the non-increased group (Table $3 ; p=0.007$ ). At the genus level, the increased group had a higher population of Actinobaculum, Dialister, and Alloprevotella than the non-increased group (Table $3 ; p<0.05$ ). At the species level, the increased group had higher populations of Neisseria sicca, Alloprevotella sp., Dialister invisus, Cardiobacterium hominis, Acinetobacter sp., Gracilibacteria (GN02) [G-1], Neisseria elongate, Actinomyces graevenitzii, Anaerolineae [G-1], Dialister pneumosintes, Haemophilus haemolyticus, Actinobaculum sp., Corynebacterium matruchotii, Prevotella pleuritidis, and Neisseria sp. than those in the non-increased group (Table 3; $p<0.05$ ). Low percentages of S. mutans in saliva were detected in both groups. In LEfSe analysis, Prevotellaceae and Veillonellaceae at the family level and Alloprevotella and Dialister at the genus level were enriched in the increased group compared with the non-increased group (Figure 3). 
Table 3. Comparison of relative abundances of bacteria between increased and non-increased groups.

\begin{tabular}{llccr}
\hline \multirow{2}{*}{ Taxonomy Level } & Non-Increased & Increased & \multirow{2}{*}{$\boldsymbol{p}$-Value ${ }^{2}$} \\
\cline { 3 - 4 } & & $(\boldsymbol{n}=\mathbf{4 1})$ & $(\boldsymbol{n}=\mathbf{1 4})$ & \\
\hline Phylum & Proteobacteria & $10.5 \pm 7.8$ & $12.2 \pm 4.3$ & 0.029 \\
Family & Prevotellaceae & $1.6 \pm 1.6$ & $2.6 \pm 2$ & 0.007 \\
Genera & Alloprevotella & $1.6 \pm 1.6$ & $2.6 \pm 2$ & 0.007 \\
& Dialister & $0.2 \pm 0.3$ & $0.3 \pm 0.5$ & 0.039 \\
& Actinobaculum & $0.1 \pm 0.1$ & $0.1 \pm 0.1$ & 0.008 \\
& Neisseria sicca; $n$. mucosa & $2.3 \pm 2.8$ & $3 \pm 2.5$ & 0.042 \\
& Alloprevotella sp. & $1.1 \pm 1.3$ & $1.7 \pm 1.7$ & 0.009 \\
& Dialister invisus & $0.1 \pm 0.3$ & $0.3 \pm 0.5$ & 0.037 \\
& Cardiobacterium hominis & $0.3 \pm 0.7$ & $0.4 \pm 0.6$ & 0.037 \\
& Acinetobacter sp. & $0.3 \pm 0.5$ & $0.5 \pm 0.5$ & 0.001 \\
& GN02 [G-1] & $0.1 \pm 0.3$ & $0.2 \pm 0.3$ & 0.031 \\
& Neisseria elongata & $0.4 \pm 0.8$ & $0.7 \pm 1$ & 0.034 \\
& Actinomyces graevenitzii & $0.1 \pm 0.1$ & $0.1 \pm 0.2$ & 0.024 \\
& Anaerolineae [G-1] & $0.1 \pm 0.1$ & $0.2 \pm 0.2$ & 0.032 \\
& Dialister pneumosintes & $0.1 \pm 0.1$ & $0.1 \pm 0.2$ & 0.015 \\
& Haemophilus haemolyticus & $0.1 \pm 0.2$ & $0.2 \pm 0.4$ & 0.019 \\
& Actinobaculum sp. & $0.1 \pm 0.1$ & $0.1 \pm 0.1$ & 0.008 \\
& Corynebacterium matruchotii & $0.1 \pm 0.1$ & $0.1 \pm 0.1$ & 0.042 \\
& Prevotella pleuritidis & $0.1 \pm 0.1$ & $0.1 \pm 0.2$ & 0.014 \\
Neisseria sp. & $0.1 \pm 0.1$ & $0.1 \pm 0.1$ & 0.021 \\
\hline
\end{tabular}

${ }^{1}$ Data are expressed as mean \pm standard deviation; ${ }^{2}$ Mann-Whitney U test.

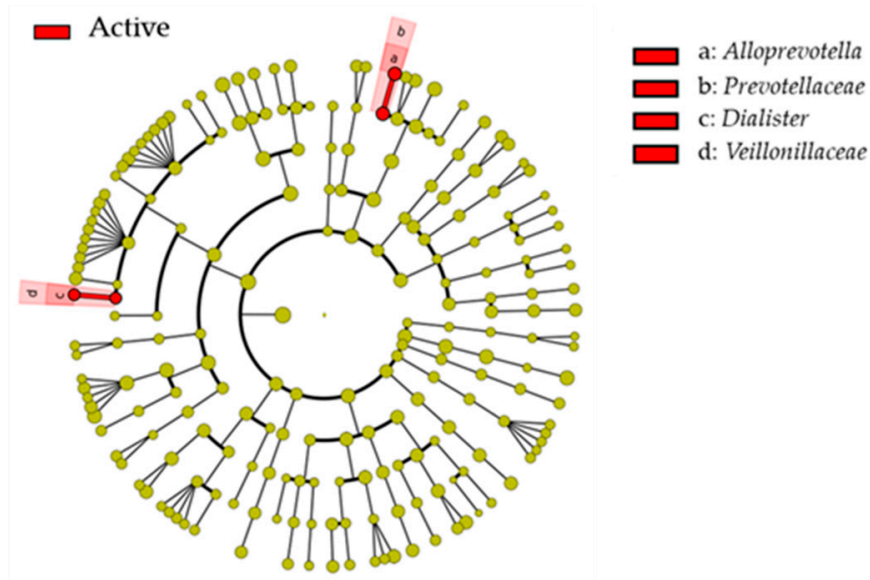

Figure 3. Linear discriminant analysis effect size (LEfSe) analysis to identify unique taxa associated with caries. Taxa at the family level were analyzed. Red areas of the cladogram were enriched in the increased group compared with the non-increased group. Linear discriminant analysis (LDA) scores $\geq 3.0$ are shown.

\section{Discussion}

Various groups have provided information about the relationship between dental caries and the oral microbiome [4,7-10,21-25]. However, there is less information about this relationship in young adults or university students. In the present 3-year cohort study, the abundance of several taxa was significantly higher in the increased group than that in the non-increased group. In addition, poor oral hygiene and caries experience at baseline were significantly associated with an increase in dental caries during university life.

In this study, the observed taxa number was not significantly different between increased and non-increased groups. Moreover, the microbial structure was similar between the two groups. Jiang et al. 
reported that the microbial composition was similar between caries progression and non-progression groups among older people [23]. The findings from our study support these results.

The percentages of several taxa were significantly higher in the increased group than those in the non-increased group. In particular, further analysis using LEfSe methods revealed families Prevotellaceae and Veillonellaceae and genera Alloprevotella and Dialister were significantly enriched in the increased group compared with the non-increased group (LDA scores $\geq 3.0$ ). Prevotellaceae was detected in carious dentin or saliva of participants with caries progression [24,25]. Furthermore, Eriksson et al. also reported that Veillonellaceae and Dialister were enriched in the saliva of patients with caries progression using the LEfSe method [20]. Alloprevotella was detected in the saliva of children with caries progression using LEfSe [4]. Using non-culture methods, non-mutans streptococci have been detected in carious dentin or saliva [26]. Furthermore, Prevotellaceae and Veillonellaceae species are known to produce acid [5,27]. These results indicate that several acid-producing bacteria or non-mutans streptococci may be associated with caries progression, which suggests new targets for preventing caries progression.

In this study, there were no significant differences in microbiome diversities between increased and non-increased groups. Previous studies exploring the oral microbiome to elucidate potential targets for caries prevention mainly targeted children with deciduous dental plaque or saliva. For example, a 2-year cohort study revealed that microbial diversity in saliva did not differ between caries progression and non-progression groups [10]. However, some cohort studies reported that salivary microbiome diversity was significantly different between participants with increased dental caries compared with those with non-increased dental caries [7,21,22]. According to these reports, whether salivary microbiome diversity is associated with caries progression remains controversial. Furthermore, few studies included young adults as subjects. A cross-sectional study exploring salivary microbiomes among families, including young adults, reported that dental caries did not impact microbial diversity [28]. While our findings support the results of this study, further cohort studies are required to investigate the relationship between microbiome diversity and an increase in dental caries.

Oral condition is also associated with caries progression. In a cohort study of adult participants in Sweden, DMFT scores of the caries progression group were higher than those in the non-progression group at baseline [29]. In addition, a previous study reported that the increased caries group had worse oral hygiene than that of the non-increased caries group [30]. Here, we demonstrated that DMFT and DI-S scores were associated with an increase in dental caries in the logistic regression analysis, supporting the results of these previous studies.

In this study, a low abundance of mutans streptococci was detected among the two groups. Mutans streptococci are traditionally recognized as the most common cause of dental caries [31]. Several studies also reported that there was no difference in the abundance of these species between caries-active and caries-free groups [7-9,32]. Furthermore, Takahashi and Nyvad proposed the "Ecological Plaque Hypothesis," wherein acid-producing bacteria, excluding mutans streptococci, are also considered to lead to an imbalance in mineralization [26]. However, we did not investigate the microbiome in dental plaques of participants with caries. Further studies are therefore required.

In this study, we used saliva samples for microbiome analysis. Saliva collection is non-invasive, simple, and effective for mass examination. Some studies reported that plaque bacteria are released into saliva [9,33], and saliva provides a niche for both anaerobic and aerobic bacteria [34]. Furthermore, saliva is a biomarker that reflects oral health and systemic condition $[35,36]$. Thus, saliva evaluation has several advantages, especially in large-scale epidemiological studies or caries screening.

Our study has some limitations. First, there may have been selection bias, given the low follow-up rate $(13.4 \%)$. In addition, the number of participants who had regular dental checkups at baseline and DI-S scores at follow-up differed between selected participants and total participants. However, increased dental caries was not related to these variables in the logistic regression analysis. Therefore, the selection bias might be small in this study. Second, all participants were recruited from among students who attended Okayama University. Therefore, our results may not be generalizable. Third, 
we could not investigate potential confounders, such as social capital [37,38] or socioeconomic status [39]. Fourth, we did not use a specific primer and probe for detecting S. mutans. Using the Human Oral Microbiome Database, we could detect the taxa using obtained sequencing reads. However, a previous study mentioned that V1-V2 or V3-V4 hypervariable regions (approximately $400-460 \mathrm{bp}$ ) is the limit for identifying detailed level of taxa [19]. Therefore, a wider region or specific primer is needed [19]. Fifth, we did not set a specific time for saliva collection; that is, saliva was collected from 09:00 to 16:00. However, we randomly selected participants and collected saliva at the last step of the general health examination. Therefore, we do not consider that eating or drinking affected salivary analysis. Finally, we could not collect saliva at baseline; however, we checked caries increment using DMFT at baseline and follow-up. Although Belstrøm et al. reported the stable condition of the salivary microbiome [40], we cannot rule out the possibility of changes in the salivary microbiome during the 3-year study period.

\section{Conclusions}

Among Japanese university students, non-mutans streptococci bacteria in saliva were associated with increased caries in this 3-year prospective cohort study. However, there were no significant differences in salivary microbiome diversity between increased and non-increased groups.

Author Contributions: Y.U.-F. drafted the manuscript. D.E. and M.M. contributed to design of the work. D.E., A.T.-T., K.K., D.F., N.T., T.K., K.F., N.S., and Y.I. contributed to data acquisition. Y.U.-F. and M.M.I. contributed to data analysis. All authors have read and agreed to the published version of the manuscript.

Funding: This work was financially supported by a Grant-in-Aid for Scientific Research (no. 15K11415) from the Ministry of Education, Culture, Sports, Science, and Technology, Tokyo, Japan.

Acknowledgments: The authors are grateful to Tetsuji Azuma (Asahi University, Japan), Mayu Yamane-Takeuchi, and Shinsuke Mizutani (Kyushu University, Japan) for data collection; and Toru Takeshita (Kyushu University, Japan) and Kazuyoshi Gotoh (Okayama University, Japan) for technical support.

Conflicts of Interest: The authors declare no conflict of interest.

\section{References}

1. Kassebaum, N.; Smith, A.G.C.; Bernabé, E.; Fleming, T.D.; Reynolds, A.E.; Vos, T.; Murray, C.J.L.; Marcenes, W.; GBD 2015 Oral Health Collaborators. Global, Regional, and National Prevalence, Incidence, and Disability-Adjusted Life Years for Oral Conditions for 195 Countries, 1990-2015: A Systematic Analysis for the Global Burden of Diseases, Injuries, and Risk Factors. J. Dent. Res. 2017, 96, 380-387. [CrossRef] [PubMed]

2. Selwitz, R.H.; Ismail, A.I.; Pitts, N.B. Dental caries. Lancet 2007, 369, 51-59. [CrossRef]

3. Schulze-Schweifing, K.; Banerjee, A.; Wade, W.G. Comparison of bacterial culture and 16S rRNA community profiling by clonal analysis and pyrosequencing for the characterization of the dentine caries-associated microbiome. Front. Cell Infect. Microbiol. 2014, 12, 164. [CrossRef]

4. Xu, L.; Chen, X.; Wang, Y.; Jiang, W.; Wang, S.; Ling, Z.; Chen, H. Dynamic alterations in salivary microbiota related to dental caries and age in preschool children with deciduous dentition: A 2-Year Follow-Up Study. Front. Physiol. 2018, 4, 342. [CrossRef] [PubMed]

5. Takahashi, N.; Nyvad, B. Ecological Hypothesis of Dentin and Root Caries. Caries Res. 2016, 50, 422-431. [CrossRef] [PubMed]

6. Aas, J.A.; Griffen, A.L.; Dardis, S.R.; Lee, A.M.; Olsen, I.; Dewhirst, F.E.; Leys, E.J.; Paster, B.J. Bacteria of dental caries in primary and permanent teeth in children and young adults. J. Clin. Microbiol. 2008, 46, 1407-1417. [CrossRef]

7. Jiang, W.; Ling, Z.; Lin, X.; Chen, Y.; Zhang, J.; Yu, J.; Xiang, C.; Chen, H. Pyrosequencing analysis of oral microbiota shifting in various caries states in childhood. Microb. Ecol. 2014, 67, 962-969. [CrossRef]

8. Jiang, S.; Gao, X.; Jin, L.; Lo, E.C.M. Salivary Microbiome Diversity in Caries-Free and Caries-Affected Children. Int. J. Mol. Sci. 2016, 17, 1978. [CrossRef]

9. Xu, H.; Hao, W.; Zhou, Q.; Wang, W.; Xia, Z.; Liu, C.; Chen, X.; Qin, M.; Chen, F. Plaque bacterial microbiome diversity in children younger than 30 months with or without caries prior to eruption of second primary molars. PLoS ONE 2014, 28, e89269. [CrossRef] 
10. Xu, H.; Tian, J.; Hao, W.; Zhang, Q.; Zhou, Q.; Shi, W.; Qin, M.; He, X.; Chen, F. Oral Microbiome Shifts from Caries-Free to Caries-Affected Status in 3-Year-Old Chinese Children: A Longitudinal Study. Front. Microbiol. 2018, 28, 2009. [CrossRef]

11. Survey of Dental Disease in Japan. 2016. Available online: https://www.mhlw.go.jp/toukei/list/62-17c.html (accessed on 1 February 2019).

12. Mizutani, S.; Ekuni, D.; Tomofuji, T.; Irie, K.; Azuma, T.; Iwasaki, Y.; Morita, M. Self-efficacy and progression of periodontal disease: A prospective cohort study. J. Clin. Periodontol. 2015, 42, 1083-1089. [CrossRef] [PubMed]

13. Greene, J.C.; Vermillion, J.R. The simplified oral hygiene index. J. Am. Dent. Assoc. 1964, 68, 7-13. [CrossRef] [PubMed]

14. World Health Organization. Oral Health Survey. Basic Methods, 4th ed.; World Health Organization: Geneva, Switzerland, 1997.

15. Mohamed, R.; Campbell LCooper-White, J.; Dimeski, G.; Punyadeera, C. The impact of saliva collection and processing methods on CRP, IgE, and Myoglobin immunoassays. Clin. Transl. Med. 2012, 1, 19. [CrossRef] [PubMed]

16. Principe, S.; Hui, A.B.; Bruce, J.; Sinha, A.; Liu, F.F.; Kislinger, T. Tumor-derived exosomes and microvesicles in head and neck cancer: Implications for tumor biology and biomarker discovery. Proteomics 2013, 13, 1608-1623. [CrossRef]

17. Yakob, M.; Fuentes, L.; Wang, M.B.; Abemayor, E.; Wong, D.T. Salivary biomarkers for detection of oral squamous cell carcinoma-current state and recent advances. Curr. Oral. Health Rep. 2014, 1, 133-141. [CrossRef]

18. Kunitomo, M.; Ekuni, D.; Mizutani, S.; Tomofuji, T.; Irie, K.; Azuma, T.; Yamane, M.; Kataoka, K.; Taniguchi-Tabata, A.; Mizuno, H.; et al. Association between Knowledge about Comprehensive Food Education and Increase in Dental Caries in Japanese University Students: A Prospective Cohort Study. Nutrients 2016, 8, 114. [CrossRef]

19. Johansson, I.; Witkowska, E.; Kaveh, B.; Lif Holgerson, P.; Tanner, A.C. The Microbiome in Populations with a Low and High Prevalence of Caries. J. Dent. Res. 2016, 95, 80-86. [CrossRef]

20. Eriksson, L.; Lif Holgerson, P.; Johansson, I. Saliva and tooth biofilm bacterial microbiota in adolescents in a low caries community. Sci. Rep. 2017, 7, 5861. [CrossRef]

21. Belstrom, D.; Fiehn, N.E.; Nielsen, C.H.; Holmstrup, P.; Kirkby, N.; Klepac-Ceraj, V.; Paster, B.J.; Twetman, S. Altered bacterial profiles in saliva from adults with caries lesions: A case-cohort study. Caries Res. 2014, 48, 368-375. [CrossRef]

22. Kim, B.S.; Han, D.H.; Lee, H.; Oh, B. Association of Salivary Microbiota with Dental Caries Incidence with Dentine Involvement after 4 Years. J. Microbiol. Biotechnol. 2018, 28, 454-464. [CrossRef]

23. Jiang, Q.; Liu, J.; Chen, L.; Gan, N.; Yang, D. The oral microbiome in the elderly with dental caries and health. Front. Cell Infect. Microbiol. 2018, 4, 442. [CrossRef] [PubMed]

24. Chhour, K.L.; Nadkarni, M.A.; Byun, R.; Martin, F.E.; Jacques, N.A.; Hunter, N. Molecular analysis of microbial diversity in advanced caries. J. Clin. Microbiol. 2005, 43, 843-849. [CrossRef] [PubMed]

25. Yang, F.; Zeng, X.; Ning, K.; Liu, K.L.; Lo, C.C.; Wang, W.; Chen, J.; Wang, D.; Huang, R.; Chang, X.; et al. Saliva microbiomes distinguish caries-active from healthy human populations. ISME J. 2012, 6, 1-10. [CrossRef] [PubMed]

26. Takahashi, N.; Nyvad, B. The role of bacteria in the caries process: Ecological perspectives. J. Dent. Res. 2011, 90, 294-303. [CrossRef] [PubMed]

27. Gross, E.; Leys, E.; Gasparovich, S.; Firestone, N.; Schwartzbaum, J.; Janies, D.; Asnani, K.; Griffen, A. Bacterial 16S sequence analysis of severe caries in young permanent teeth. J. Clin. Microbiol. 2010, 48, 4121-4128. [CrossRef] [PubMed]

28. Foxman, B.; Luo, T.; Srinivasan, U.; Ramadugu, K.; Wen, A.; Goldberg, D.; Shedden, K.; Crout, R.; McNeil, D.W.; Weyant, R.; et al. The effects of family, dentition, and dental caries on the salivary microbiome. Ann. Epidemiol. 2016, 26, 348-354. [CrossRef] [PubMed]

29. Söderström, U.; Johansson, I.; Sunnegårdh-Grönberg, K. A retrospective analysis of caries treatment and development in relation to assessed caries risk in an adult population in Sweden. BMC Oral. Health 2014, 17, 126. [CrossRef] 
30. Tafere, Y.; Chanie, S.; Dessie, T.; Gedamu, H. Assessment of prevalence of dental caries and the associated factors among patients attending dental clinic in Debre Tabor general hospital: A hospital-based cross-sectional study. BMC Oral. Health 2018, 18, 119. [CrossRef]

31. Van Houte, J. Role of micro-organisms in caries etiology. J. Dent. Res. 1994, 73, 672-681. [CrossRef]

32. Inquimbert, C.; Bourgeois, D.; Bravo, M.; Viennot, S.; Tramini, P.; Llodra, J.C.; Molinari, N.; Dussart, C.; Giraudeau, N.; Carrouel, F. The oral bacterial microbiome of interdental surfaces in adolescents according to carious risk. Microorganisms 2019, 7, 319. [CrossRef]

33. Takeshita, T.; Kageyama, S.; Furuta, M.; Tsuboi, H.; Takeuchi, K.; Shibata, Y.; Shimazaki, Y.; Akifusa, S.; Ninomiya, T.; Kiyohara, Y.; et al. Bacterial diversity in saliva and oral health-related conditions: The Hisayama Study. Sci. Rep. 2016, 24, 22164. [CrossRef] [PubMed]

34. Chattopadhyay, I.; Verma, M.; Panda, M. Role of Oral Microbiome Signatures in Diagnosis and Prognosis of Oral Cancer. Technol. Cancer Res. Treat. 2019, 18, 1533033819867354. [CrossRef]

35. Patil, P.B.; Patil, B.R. Saliva: A diagnostic biomarker of periodontal diseases. J. Indian Soc. Periodontol. 2011, 15, 310-317. [CrossRef]

36. Lorenzo-Pouso, A.I.; Pérez-Sayáns, M.; Bravo, S.B.; López-Jornet, P.; García-Vence, M.; Alonso-Sampedro, M.; Carballo, J.; García-García, A. Protein-Based Salivary Profiles as Novel Biomarkers for Oral Diseases. Dis. Mark. 2018, 7, 6141845. [CrossRef] [PubMed]

37. Fisher-Owens, S.A.; Gansky, S.A.; Platt, L.J.; Weintraub, J.A.; Soobader, M.J.; Bramlett, M.D.; Newacheck, P.W. Influences on children's oral health: A conceptual model. Pediatrics 2007, 120, 510-520. [CrossRef] [PubMed]

38. Furuta, M.; Ekuni, D.; Takao, S.; Suzuki, E.; Morita, M.; Kawachi, I. Social capital and self-rated oral health among young people. Community Dent. Oral. Epidemiol. 2012, 40, 97-104. [CrossRef]

39. Slade, G.D. Epidemiology of dental pain and dental caries among children and adolescents. Community Dent. Health 2001, 18, 219-227.

40. Belstrøm, D.; Holmstrup, P.; Bardow, A.; Kokaras, A.; Fiehn, N.E.; Paster, B.J. Comparative analysis of bacterial profiles in unstimulated and stimulated saliva samples. J. Oral. Microbiol. 2016, 16, 30112. [CrossRef]

(C) 2020 by the authors. Licensee MDPI, Basel, Switzerland. This article is an open access article distributed under the terms and conditions of the Creative Commons Attribution (CC BY) license (http://creativecommons.org/licenses/by/4.0/). 